\title{
An IIoT Edge Environment as a Main Support to a 3D Reconstruction Virtualization Application
}

\author{
Leya Badat ${ }^{1,3}$, Vinicius Vidal ${ }^{2}$, Laércio Pioli ${ }^{3}$, Jean-Francois Mehaut ${ }^{1}$, Leonardo \\ Honorio ${ }^{2}$, Mario A.R. Dantas ${ }^{3}$ \\ ${ }^{1}$ Université Grenoble Alpes, CNRS, Inria, GrenobleINP, LIG, Grenoble, France \\ ${ }^{2}$ Department of Electrical Engineering Federal, University of Juiz de Fora, Juiz de Fora, \\ MG - Brazil \\ ${ }^{3}$ Department of Computer Science Federal University of Juiz de Fora \\ Juiz de Fora, MG - Brazil
}

\begin{abstract}
leya.badatelive.fr, jean-francois.mehauteuniv-grenoble-alpes.fr, vinicius.vidal@engenharia.ufjf.br, leonardo.honorio@ufjf.edu.br, \{laerciopioli,mario.dantas\}@ice.ufje.br
\end{abstract}

\begin{abstract}
This paper describes an experimental research work, which was conducted to gather different types of cameras and computer node, as IIoT (Industrial IoT) devices, to produce support to a digital transformation with a $3 D$ reconstruction virtualization for a real engineering application. The computational approach considered was a heterogeneous edge computing environment. In this environment, different cameras and computer node architectures, collaborate as heterogeneous IIoT processing elements, to provide a better, and as fast as possible, images to a virtualization project. The challenge and complexity related to software packages orchestration, from these IIoT devices, are also reported.
\end{abstract}

Resumo. Este artigo descreve um trabalho experimental de pesquisa que foi realizado para agregar diferentes tipos de câmeras e um nodo computacional, como nodos IoT Industriais (IIoT). O objetivo foi produzir um suporte para uma transformação digital para uma virtualização baseada em reconstrução $3 D$ para um aplicativo real de engenharia. A abordagem computacional considerada foi um ambiente heterogêneo, com dispositivos IIoT, para computação nas bordas. Neste ambiente, diferentes câmeras $e$ o nodo computacional, com arquiteturas distintas, colaboraram como elementos IIoT para fornecer melhores imagens, o mais rápido possível, para um projeto baseado em virtualização. Os desafios e as complexidades relacionados à orquestração dos pacotes de software, desses dispositivos IIoT, são também reportados.

\section{Introduction}

China is the world's largest producer of hydropower and accounted for nearly half of global added installed capacity, at $9.1 \mathrm{GW}$. It is followed by Brazil (3.4 GW), India (1.9 GW), Portugal (1.1 GW) and Angola (1.0 GW) [Hydropower, 2020]. Energy management is one of the main pillars of Industry 4.0. The previous 
numbers illustrate the challenge to work with this Industry 4.0 segment, which also represents $64 \%$ of energy produced in Brazil. Furthermore, the distributed geographical area of the scenario is around 8.5 millions of square kilometres.

Virtual and/or augmented reality systems are usually adopted as a basis to enhance engineering projects efforts. One interesting mechanism to reproduce three-dimensional objects and scenarios in virtual environments is to develop a reconstruction software application. As observed in work presented in [Silva, 2019], this option is faster and less costly alternative in specialized labour. Especially, in comparison to ordinary form of generating these objects, usually, through graphic design with a specialized software. However, requirements are becoming more complex, especially to IoT software application developers, as observed in the work [RC Motta et al., 2018].

On the other hand, the number of devices required in these reconstruction processes could be very large and with different type of architectures. Therefore, an orchestration is required to gather and to produce synchronized differentiated results from these different architecture nodes. As mention in the case study presented in [Gopika et al., 2018], edge computing platforms can be classified as (i) resource-rich servers deployed at the edge, (ii) heterogeneous edge nodes and edge-cloud federation, and (iii) based upon their classes and architectures. Heterogeneous edge nodes are interesting architectures to tackle issues where the configuration requires different kind of IoT devices to provide successful results. This is the case example when devices, such as cameras, are utilized to gather data, to process, and provide an accurate information to an application.

Our work is one experimental element of collaboration in a large real project, in the electricity industry segment, called as a Virtual Project [Silva, 2019]. The project is a real effort in the Brazilian electricity industry that targets excellence in assisted maintenance and people training, considering the paradigm of Industry 4.0. Therefore, it is important to mention that in this research work we considered devices as IIoT (Industrial IoT) equipment, because in the end of the day, they will be utilized in an industrial environment. In this paper we present an experimental research contribution work, which is characterized by heterogeneous IIoT edge nodes, as a computational environment, to provide data to a 3D reconstruction application. Our experiments show interesting set of aspects and issues considering this heterogeneous edge configuration.

The paper is organized as follows. In section II, we present some theoretical concepts related to technologies used in this research work. The Virtual Project, a 3D reconstruction software application, and related work are described in section III. In section IV, it is illustrated the edge experimental environment and results. Finally, in section $\mathrm{V}$, we present conclusions and future works.

\section{Theoretical Concepts}

This section presents some concepts related to ROS, Point Cloud Library (PCL), odometry and a heterogenous edge platform, because these elements represent the base of the experimental environment.

\subsection{ROS}

Robot Operating System (ROS) [ROS, 2020] is an open source robot software development platform. It is a meta-operating system that can run on one or more computers and provides several features. Examples of such features are hardware abstraction, low-level device control, implementation of commonly used features, 
messaging between the processes and management of the installed packages. Its collection of tools, libraries and conventions aim to simplify the task of people that want to write complex robotic behavior. There are five main functionalities: an architecture allowing inter-process and inter-machine communication; a system of recording data into what are called bagfiles, and of replaying them; a testing sytem; a parameter server; a simulator called Gazebo.

The concept of file system in ROS is organized into packages, that can contain libraries, executables, scripts, etc. Those packages are described by manifests, which are XML files that serve to define the possible dependencies between different packages and providing meta information about the package, such as its version.

ROS offers a flexible communication architecture. The processes are called nodes and each node can communicate with other topics. The connection between the nodes is managed by a master. The following process can help the understanding:

- Node 1 tells the master that it wants to share data on Topic A.

- Node 2 tells the master that it needs to access data on Topic A.

- A connection is created on Topic A between Node 1 and Node 2.

- Node 1 can send data on Topic A and Node 2 can collect them.

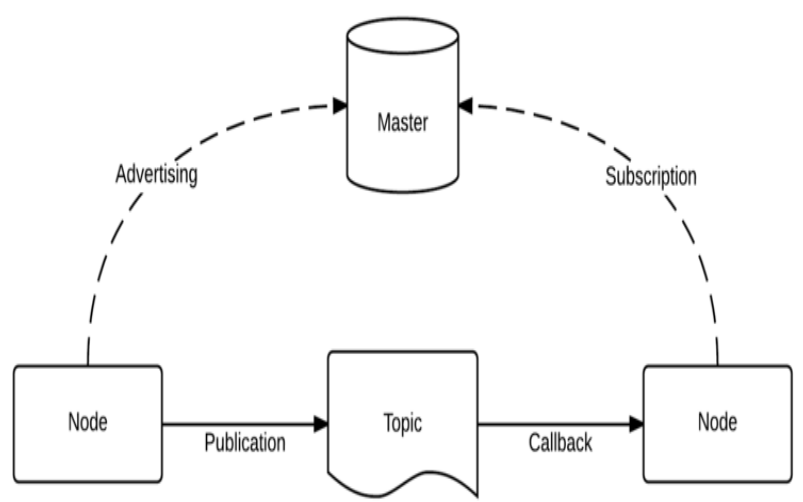

Figure 1. Master's operation in ROS.

A node that publishes data is called a publisher and a node that subscribes to data is called a subscriber. A node can be both publisher and subscriber. The messages sent on the topics are standardized in a specific ROS data type, which makes the system extremely flexible.

\subsection{Point Cloud Library (PCL)}

Point clouds are sets of data points in space. It is usually produced by $3 \mathrm{D}$ sensors that are measuring a large amount of points on the surfaces of objects that they are analysing.

Point Cloud Library (PCL) [PCL, 2020] is an open source library of algorithm used for tasks involving point cloud, or 3D geometry processing. PCL is really fitted to be used in ROS, especially because a specific ROS data structure that can represent point clouds: pcl::PointCloud $<\mathrm{T}>$. This is the core point cloud class in PCL and it can be a template on any of the usual Point types. In the point cloud processing nodes, it is better to work with this template class that help define each individual point as an object. 


\subsection{Odometry}

Odometry is the use of data collected from motion sensors in order to estimate changes in position over time. It is sensitive to errors, because of the integration of velocity measurements over time to provide an estimation of the position. Odometry requires rapid accurate data gathering, and a precise instrument calibration. In this project, a special kind of odometry is used due to the cameras being the sensors, the visual odometry. It is the process of determining equivalent odometry information using sequential camera images to estimate the distance that has been traveled.

\subsection{Heterogeneous Edge Platform}

Following the definition found in [Gopika et al., 2018], edge computing platforms can be classified as (i) resource-rich servers deployed at the edge. (ii) heterogeneous edge nodes and edge-cloud federation, and (iii) based upon their classes and architectures.

In the case of heterogeneous nodes at the edge, including the end-devices themselves differs from the previous scenarios, because this classification covers a diverse set of computing resources, such as a fog platform which has the characteristics of a high virtualized system of heterogeneous nodes. This heterogeneity can be also considered for the wireless connectivity aspects. In other words, different devices in a fog platform fashion shape are orchestrated to process data for applications.

\section{The Virtual Project}

The Virtual Project is a reconstruction software application being developed at the GRIn [GRIn, 2020], an Inerge lab [Inerge, 2020], with some collaboration from researcher members from the computer science knowledge group [NenC, 2020]. The project involves professors and students from undergraduate to postgraduate level, from engineering and computer science. Foreigners students visiting our labs also contribute with their effort, which is the case stated in this paper with the collaboration with Grenoble Alpes University.

The primary goal of the project is to support a supervision of electricity substations equipment, i.e. to design an approach which can provide a solution for equipment maintenance and crew training considering in an Industry 4.0 scenario. The Virtual Project is based upon the technology and advances in the computer vision area, providing a reconstruction of an equipment and/or an environment. Several techniques and equipment are experimented and tested to fulfill this purpose. Examples of equipment comprises image and laser data processing, as much as onboard and parallel programming for a low and high level of information processing.

The technological paradigm of the project effort can be understanding as the utilization of virtual reality glasses and smartphones/tablets after the reconstruction of an equipment or environment. Therefore, professionals can be trained for dangerous situations with no need for equipment and real-life simulations. The energy generation and transmission industries can benefit by this type of technology. Large equipment makes it hard to keep track of maintenance techniques, and transmission faults situation must be dealt in the least amount of time, due to high penalty values from the government.

In the Virtual Project the first moment is characterized by the environment and equipment scanned. Next, both are placed and worked in a virtual and augmented reality application. Afterwards, a 3D point cloud is gathered with field scanners built and programmed in a laboratory with the latest sensor technology in the market. The sensor 
calibration must be accurate and adaptive to work in the most variety of environments. Once the point cloud is acquired, it must be manipulated to create a mesh and a textured model.

Therefore, the whole environment or dedicated equipment can be inserted in the application. In this point, images are gathered from the environment, then computer graphics applications are utilized to create 360 degrees view of the scene from that point. Virtual reality applications can place the employee in front of an equipment that could be miles away. However, its data can be monitored from anywhere as in a realworld situation.

\subsection{Related Work}

In this section we present related work in two dimensions. The first dimension highlights research related to the Edge-Fog-Cloud computing environments. In the other hand, the second dimension illustrates examples to virtualization and 3D reconstructions.

In [Kaur et al.,2018], the authors present an effort in edge computing, considering IIoT environment, with a special focus in an SDN approach. The work shown in [Sabella, 2019] tackles the challenge related to the development of the software for multi-access edge computing. A study case of an application on a fog-cloud cooperation is presented in [Dantas, Bogoni, Filho, 2020]. Finally, in [Thomas, 2019] it is illustrated aspects related to the future technology for IoT, considering edge cloud computing.

Considering the dimension of virtualization and reconstruction, in the work presented in [H. Qing, 2010], it is stated an effort in utilizing virtual reality for a previous analysis for mechanical equipment maintenance, targeting the time reduction and cost of the maintenance. Spacecrafts reconstruction and the 3D models which stores relevant historical information are described in the [D. Shcherbinin,2017]. Authors from the work shown in [Stumberg et al.,2017] present a research which considers from monocular SLAM to autonomous drone exploration targeting to map and locate obstacles and reconstruction without texture. On the other hand, [Schonberger and Frahm, 2016] describe an approach based upon structure from motion, based on matching features and SIFT descriptors. In [Whelan et al.,2015] a contribution characterized by tackling dense SLAM in real time, focusing on the map, based on surface elements and deformation graph.

\section{IIoT Edge Experimental Environment and Results}

In this section, we presented our experimental research proposal work, particularly aspects of the heterogeneous edge computing environment, and experiments results. This effort represents one contribution to the Virtual Project. The contribution of the present paper is an experimental research (figure 2 illustrates three components), which was based in previous works from our group presented in [Silva, 2019] and [L. Silva et al., 2018], from where we followed one proposed future work direction. The direction selected was the composition of a new heterogeneous environment formed by cameras and the utilization of an edge computing node, such as those IIoT devices from figure 2. This research was developed in parallel with another collaborative team [Decamps et al., 2020]. Their focus was in the full integration with the Jetson TX-2. Therefore, specific results utilizing the Jetson TX-2 can be seen in [Decamps et al., 2020]. 


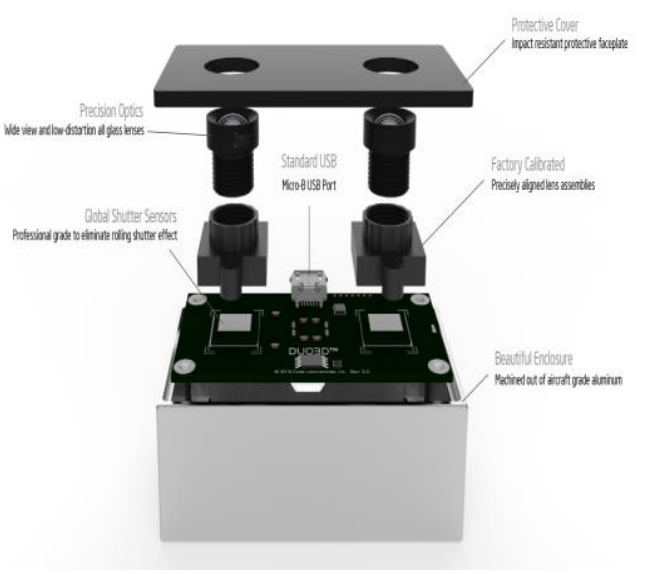

(a) Duo MC câmera.

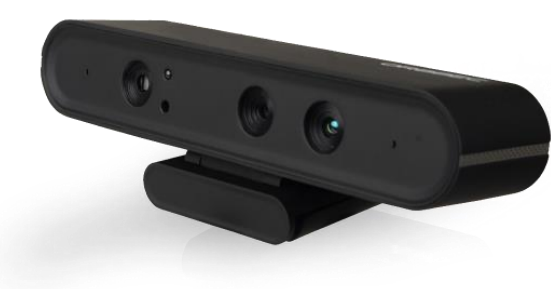

(b) Orbbec Astra 3D câmera.

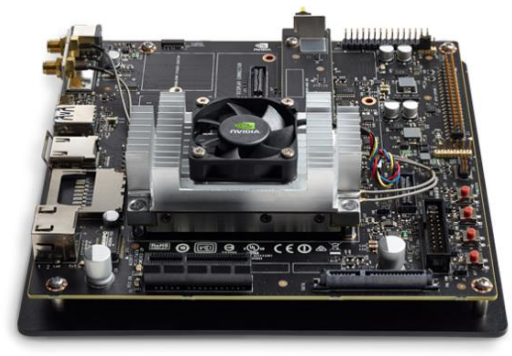

(c) Nvidia Jetson TX-2.

Figure 2. The heterogeneous IloT devices.

\subsection{DUO MC camera}

The first camera. which we had the opportunity to work with was the DUO MC [Duo $\mathrm{MC}, 2020]$. This is a tiny and compact configurable stereo camera, shown in figure 2(a), measuring depth at high speed and precision in a range between 0.08 and 1.91 meters. This camera is compatible on all platforms, and the developer's website is helpful when it comes to make all the needed installations. There is no information about colors given by this camera. Important to observe that this camera is one component in the heterogeneous edge scenario, with its own software characteristics. As a result, we early understand the required effort as a software engineering's related to the development of a contribution in an IIoT environment. This challenge point it is reported in [Motta, Oliveira and Travassos, 2018], in a research called as on challenges in IoT engineering software systems.

\subsection{Orbbec Astra camera}

The second camera, which we had to work with was the Orbbec Astra. This device can measure both color and depth, this duo to an infrared laser. However, the range is shorter in comparison to the DUO camera (between 0.6 and 0.8 meters). However, this is not a problem since we are not using it on long distances. This aspect highlights the importance of our experimental contribution, i.e. in the orchestration of the different heterogeneous devices with different characteristics.

\subsection{Nvidia Jetson TX-2}

The Nvidia Jetson TX-2 [Jetson, 2020] is the component which brings features of highperformance computing and AI at the edge. It features a variety of standard hardware 
interfaces that make it easy to integrate it into a wide range of products and form factors. The features of this computer are processor ARM64 quad-cores, RAM 8GB DDR4, 256-core Pascal GPU, 5.5-19.6VDC input power (7.5W under typical load), Ubuntu 16.04-Tegra. As it was mention before, in [Decamps et al., 2020] it is possible to obtain some ideas from this device inside the Virtual Project.

\subsection{Heterogeneous Edge Environment}

Figure 3 shows the heterogenous edge computing environment, where the previous described three devices work in unique orchestration. As we mention early, it was the infrastructure for two collaboration teams inside the engineering.

Figure 3 illustrates the interaction of the Astra camera, Duo camera with the computer node. The first camera acts as the calibration of point cloud node. The other camera performs functions as an ORB-SLAM 2 node. Finally, the compute node executes the function of registering and filtering, and then store the output registered point cloud.

In this project, our research work was to synchronize both cameras to get the best of both, because Astra camera the depth measurement is very accurate, mainly because of the facilities of color and depth. On the other hand, the DUO MC camera is not the best device with characteristics to provide depth measurement. However, it provides interesting information about odometry.

\subsubsection{TF Software Package}

The robotic system which we were working on was composed of two cameras, which means that we had to keep track of two 3D coordinates frames that changed over time. In order to do that, we utilized the tf package [tf, 2020], version tf2.

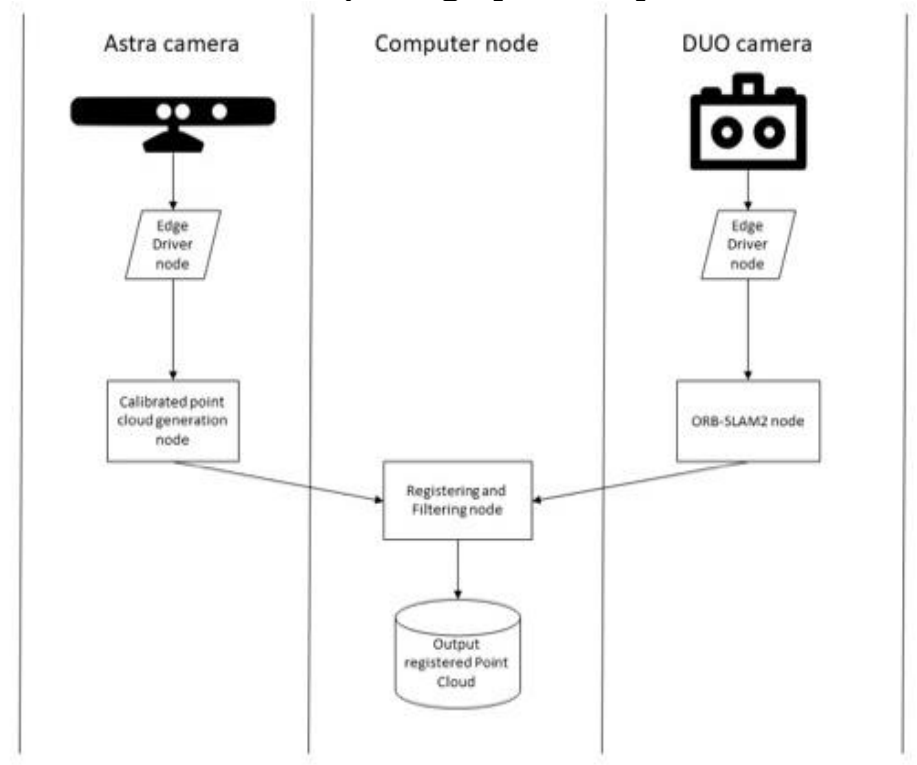

Figure 3. The heterogeneous edge computing environment.

As it is presented in [tf,2020]: "tf is a package that lets the user keep track of multiple coordinate frames over time. tf maintains the relationship between coordinate frames in a tree structure buffered in time". In addition, allows the user to transform points, vectors, etc between any two coordinate frames at any desired point in time".

In this scenario, we know the position of our two cameras compared to the world, which these cameras are in. However, because we want to synchronize those IIoT 
devices, we need to see their positions from each other. The tf software package helps to realize this transformation. Transforms are here read as homogeneous transforms, which are $4 \times 4$ matrixes.

Figure 4 shows cameras interactions, we have the transform from world to camera 1 (C1) called TWC1 and the transform from world to camera 2 (C2) called TWC2. We want to transform from $\mathrm{C} 1$ to $\mathrm{C} 2$ frames, i.e. $\mathrm{T} \mathrm{C} 1 \mathrm{C} 2$.

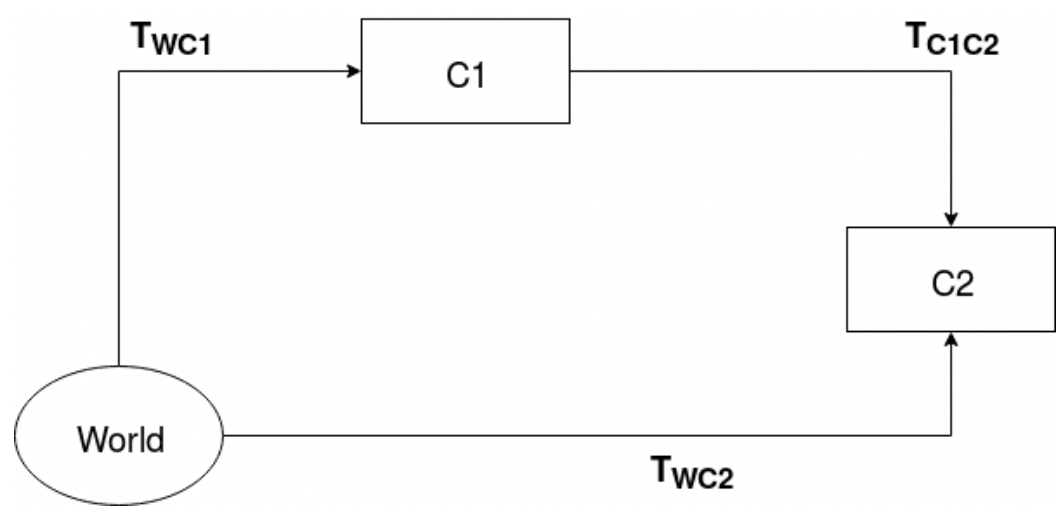

Figure 4. Cameras interactions.

If we move from World to $\mathrm{C} 1$, then to $\mathrm{C} 2$, we have the same result as if we moved straight to $\mathrm{C} 2$ from World. Therefore:

$$
\mathrm{T} \mathrm{WC} 2=\mathrm{TWC} 1 * \mathrm{~T}_{\mathrm{C}} 1 \mathrm{C} 2
$$

We want to isolate $\mathrm{T}_{\mathrm{C} 1 \mathrm{C} 2}$ in the previous equation. It can be done by multiplying $\mathrm{T}^{-1}{ }_{\mathrm{WC} 1}$ from the left on both sides of the equation.

$$
\mathrm{T}^{-1} \mathrm{WC} 1 * \mathrm{~T}_{\mathrm{WC} 2}=\mathrm{T}^{-1} \mathrm{WC1} * \mathrm{~T}_{\mathrm{WC} 1} * \mathrm{~T}_{\mathrm{C} 1 \mathrm{C} 2}
$$

OR

$$
\mathrm{T}^{-1} \mathrm{WC} * \mathrm{~T}_{\mathrm{WC} 1}=\mathrm{I}_{4}
$$

AND

THEN

$$
\mathrm{T}^{-1} \mathrm{WC} 1=\mathrm{T}_{\mathrm{CW} 1}
$$

$$
\mathrm{T}_{\mathrm{CW} 1} * \mathrm{~T}_{\mathrm{WC} 2}=\mathrm{T}_{\mathrm{C} 1 \mathrm{C} 2}
$$

that is the formula we must use in order get the position from one camera (for example, the DUO MC) compared to the other (in this case the Orbbec Astra).

\subsubsection{RGB image with Point Cloud}

In order to match RGB image with point cloud, it is required to have the calibration information for the RGB camera (the Astra) and to know the relative transform between the latter and the sensor frame of the DUO camera. For the calibration information, we had to adapt a tutorial existing for another camera close to the Astra: the Kinect.

We employed the OpenCV. First, we must transform the point cloud, afterwards it is in the optical coordinate frame of the RGB camera. Afterwards, the project the 3D points into 2D pixel locations within the image of the RGB camera, thanks to the function present in the OpenCV library, cv::projectPoints.

However, this only reconstruct a specific part of the environment. In order to create a space, we must accumulate the point clouds, based on their relative position. 
Our contribution could be understood as both Orbbec Astra and DUO MC cameras were used in synchronization. Therefore, we were able to gather the information, which was published on the depth, odometry and RGB topics. As a result, it was possible to create an accumulated point cloud, representing a part of the environment which we were working in, i.e. the laboratory. Targeting to illustrates our efforts, figure 5 shows the interface of Ros Visualization (rviz) while running the DUO MC.

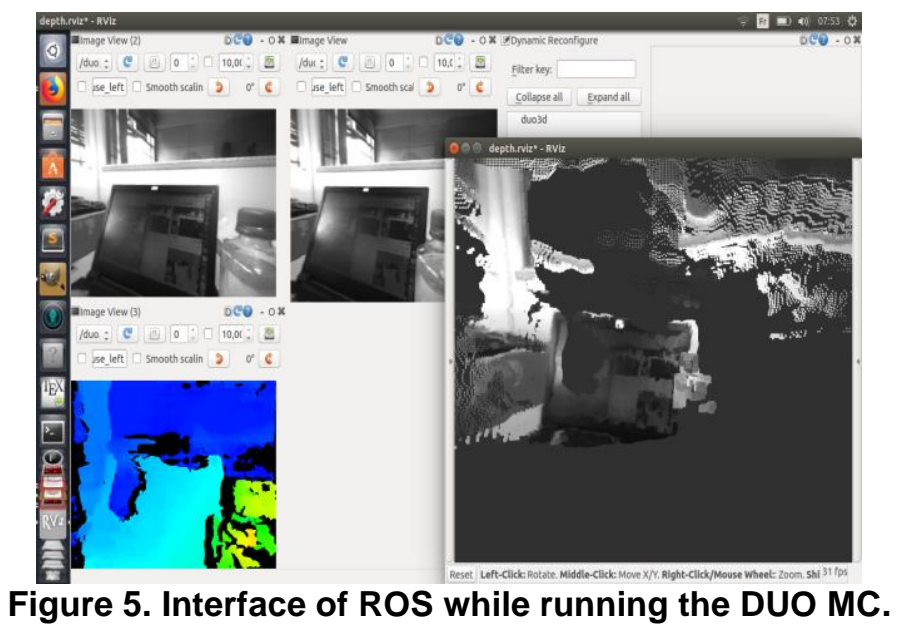

On the other hand, figure 6 brings the idea of the calibration phase of the DUO MC. These two figures show a small part of our experimental effort.

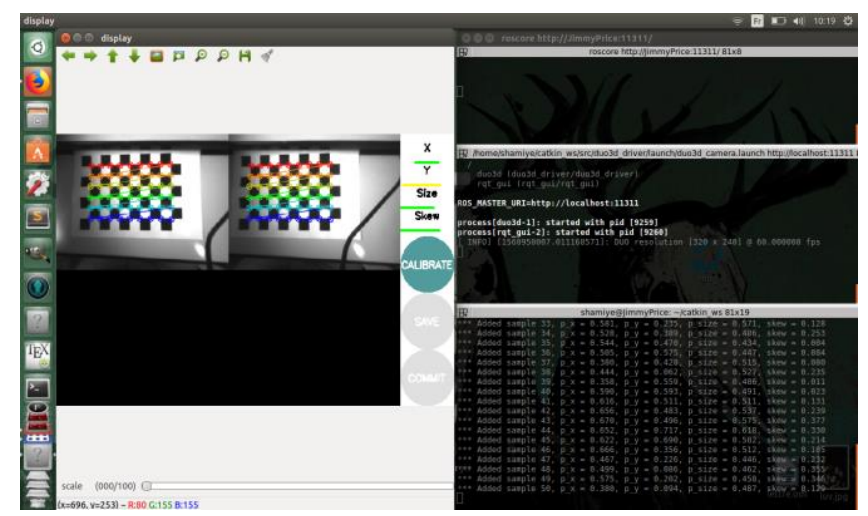

Figure 6. Calibration phase of the DUO MC.

Principal shapes and colors can be identified on figure 7, from the laboratory scenario. Where, red brick wall, turned-off computer screen, blue chairs, shape of tables. The data used to produce this result were not gathered and processed in real time. In other words, it was extracted from a file, previously registered, and it is possible that some of them were corrupted. 


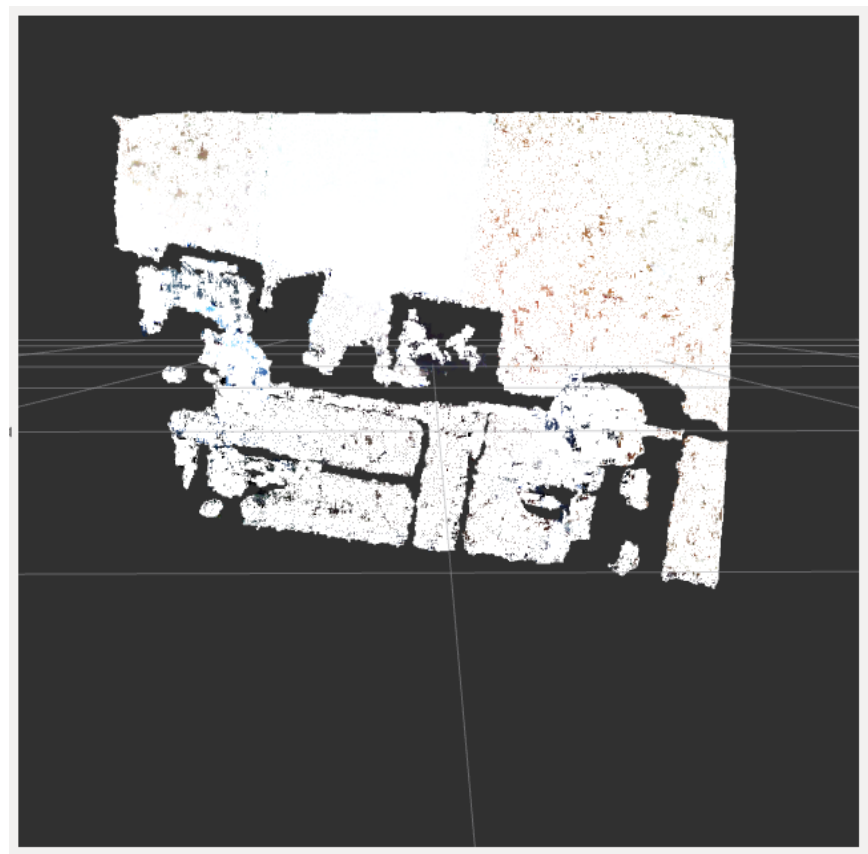

Figure 7. View of the laboratory through accumulated point clouds.

The experimental result from figure 7 , it is not the ideal one, because shapes are rough, and the color schemes essentially consist in sparse pixels. However, this issue may be tackled by using another algorithm when it comes to the point cloud processing. In addition, the local computer utilized during these experiments demonstrated to be not suitable. This aspect is a relevant experimental indication to the Virtual Project, which show the necessary selection of computing facilities to the remote location, specially when utilizing two state-of-the arts IIoT devices, in this case the two cameras.

\section{Conclusions and Future Work}

In this paper we presented an interesting experimental research, which was an element in a large scenario of an engineering project, with the focus is a $3 \mathrm{D}$ reconstruction. The Virtual Project is a reconstruction software application being developed at an electrical engineering lab with some collaboration from research members from the computer science area.

Therefore, the experimental research work conducted presented efforts to gather different types of cameras and computer node, as IIoT (Industrial IoT) devices, to support the engineering application. The computational approach adopted was a heterogeneous edge computing environment. Where different cameras and computer node architectures collaborate, as heterogeneous IIoT processing elements to provide a better and fast image to the virtualization project. The challenge and complexity related to software packages orchestration from these IIoT devices were found and reported.

As a future work, the group is planning to tackle issues and limitation found, during our experiments, adopting a fog environment infrastructure. It is also another direction a study to report in details the challenge of the IoT systems in terms of software. This study may help new developers in understanding how devices could be better orchestrated in terms of software packages and limitation from those. 


\section{Acknowledgments}

The work reported in this paper was performed as part of an interdisciplinary research and development project undertaken by UFJF. The authors acknowledge the financial funding and support of the following companies: TBE and EDP under supervision of ANEEL - The Brazilian Regulatory Agency of Electricity. Project number PD PD02651-0013/2017. In addition, authors thank the sponsor from INESC Brasil, University of Grenoble Alpes and Brazilian National Research Council (CNPq).

\section{References}

Astra, https://orbbec3d.com/, accessed in February 2020.

D. Shcherbinin, "Virtual reconstruction and $3 \mathrm{~d}$ visualization of vostok space- craft equipment," in Engineering Technologies and Computer Science (EnT), 2017 International Workshop on, pp. 56-58, IEEE, 2017.

Dantas, M.A.R., Bogoni, P.E. and Filho, P.J.d.F. (2020) 'An application study case tradeoff between throughput and latency on fog-cloud cooperation', Int. J. Networking and Virtual Organisations, Vol. 23, No. 3, pp.247-260.

Decamps at al., A Heterogenous Edge Computing Platform Case Study Supporting a Virtual Scenario Application, submitted to ISCC 2020, 2020

DuoMC, https://duo3d.com/product/duo-mc-lv1, accessed in February 2020.

Eduardo Camilo Inácio, Jorji Nonaka, Kenji Ono, Mario A. R. Dantas, Fumiyoshi Shoji: Characterizing $\mathrm{I} / \mathrm{O}$ and Storage Activity on the $\mathrm{K}$ Computer for PostProcessing Purposes. ISCC 2018: 730-735.

Gopika Premsankar, Mario Di Francesco, Tarik Taleb, "Edge Computing for the Internet of Things: A Case Study", IEEE Internet of Things Journal , vol. 5, Issue: 2, pp 1275 - 1284, April 2018.

Grin, https://www.ufjf.br/inerge/institucional/laboratorios/grin/, accessed in February 2020.

H. Qing, "Research and application of virtual reality technology in mechanical maintenance," in International Conference on Advanced Technology of Design and Manufacture (ATDM 2010), p. 256 - 258, IET, 2010.

Hydropower, https://www.hydropower.org/country-profiles/brazil, accessed in 2020.

Inerge, https://www.ufjf.br/inerge/, accessed in February 2020.

Jan Erik Solem. Programming Computer Vision with Python. O'Reilly, O'Reilly Media, Inc., 1005 Gravenstein Highway North, Sebastopol, CA 95472, 2012.

Jetson, https://developer.nvidia.com/embedded/jetson-tx2, accessed in February 2020.

Jetson Hacks, JetsonHacks.com, accessed in February 2020.

Kaur, Kuljeet, et al. "Edge computing in the industrial internet of things environment: Software-defined-networks-based edge-cloud interplay." IEEE communications magazine 56.2 (2018): 44-51.

L. Silva, V. Vidal, M. Silva, M. Santos, A. Carvalho, A. Cerqueira, L. Honório, H. Rezende, J. Ribeiro, A. Pancoti, et al., "Automatic recognition of electrical grid elements using convolutional neural networks," in 2018 22nd International Conference on System Theory, Control and Computing (ICSTCC), pp. 822-826, IEEE, 2018. 
NenC, http://www.ufjf.br/nenc/, accessed in Februry 2020.

Pointclouds, http://pointclouds.org, accessed in February 2020.

RC Motta, KM de Oliveira, GH Travassos, "On challenges in engineering IoT software systems"'SBES '18: Proceedings of the XXXII Brazilian Symposium on Software Engineering Pages 42-51, September 2018.

ROS, http://www.ros.org/, accessed in February 2020.

Sabella, Dario, et al. "Developing software for multi-access edge computing." ETSI (2019).

Silva, Luiz Augusto Zillmann, Reconstruction with multiple cameras and distributed system under the fog paradigm, Msc Dissertation, Electrical Engineering Department, UFJF, https://www.ufjf.br/ppee/2019/02/21/defesa-de-dissertacao-demestrado-luiz-augusto-zillmann-da-silva/, 2019.

Schonberger, J. L., Frahm J.-M., "Structure-from-motion revisited," in Proceedings of the IEEE Conference on Computer Vision and Pattern Recognition, pp. 4104-4113, 2016.

Stumberg, L. von, V. Usenko, J. Engel, J. Stückler, and D. Cremers, "From monocular slam to autonomous drone exploration," in Mobile Robots (ECMR), 2017 European Conference on, pp. 1-8, IEEE, 2017.

T. Masood and J. Egger. Augmented reality in support of industry 4.0 - implementation challenges and success factors. Robotics and Computer Integrated Manufacturing, 58:181_195, 2019.

tf, http://wiki.ros.org/tf, accessed in March 2020.

Thomas, Lucia Agnes Beena. "Edge Cloud: The Future Technology for Internet of Things." Novel Practices and Trends in Grid and Cloud Computing. IGI Global, 2019. 107-131.

Whelan T., M. Kaess, H. Johannsson, M. Fallon, J. J. Leonard, and J. McDonald, "Realtime large-scale dense rgb-d slam with volumetric fusion," The International Journal of Robotics Research, vol. 34, no. 4-5, pp. 598-626, 2015. 